\title{
HIV Seroprevalence Among Homeless Patients Admitted to a Psychiatric Inpatient Unit
}

\author{
Maureen Empfield, M.D., Francine Cournos, M.D., Ilan Meyer, M.A., M.Phil., \\ Karen McKinnon, M.A., Ewald Horwath, M.D., Michael Silver, M.D., \\ Howell Schrage, M.D., and Richard Herman, M.A.
}

\begin{abstract}
Obiective: This study was conducted to determine the seroprevalence of HIV-1 antibodies among hospitalized homeless mentally ill patients. Method: From December 1989 through May 1991 the authors collected discard blood samples from patients consecutively admitted to a psychiatric unit designated for the care of severely mentally ill persons removed from the streets of New York City. The blood samples were tested for HIV-1 antibodies, and the results were analyzed for associations with age, gender, ethnicity, male homosexual activity, and use of injected drugs. Results: The HIV seroprevalence was 6.4\% (13 of 203 samples). Patients between ages 18 and 39 accounted for $51.2 \%$ of the admissions and $84.6 \%$ of the 13 positive results, a seroprevalence of $10.6 \%$ for this subsample. Patients under age 40 were more than six times as likely to test positive for HIV antibodies as those 40 or over. Ethnicity did not predict seropositivity. Women were as likely as men to be infected. Although clinicians had noted high-risk behavior on the charts for only three (23.1\%) of the 13 positive cases, a recorded history of use of injected drugs was associated with a 6.5-fold greater risk of HIV seropositivity. Conclusions: One in every 16 patients admitted to the special unit was HIV positive. Age under 40 and use of injected drugs were strongly associated with seropositivity. Because information on high-risk behavior was infrequent, the reasons for younger patients' greater risk are unclear. The homeless mentally ill require outreach efforts to reduce the risk of acquiring or transmitting HIV.

(Am J Psychiatry 1993; 150:47-52)
\end{abstract}

$\mathrm{T}$ he effects of the HIV epidemic on persons who are homeless and mentally ill have not been adequately investigated. Few reports on the prevalence of HIV infection among the homeless have been published, and there have been none on the prevalence among the homeless mentally ill.

Estimating the prevalence of HIV among persons who are both mentally ill and homeless is difficult, as they constitute a "hard to reach" population (1). The homeless mentally ill are likely to be chronically disaf-

Received Nov. 27, 1991; revision received April 13, 1992; accepted May 7, 1992. From Creedmoor Psychiatric Center, Washington Heights Community Service, and New York State Psychiatric Institute, New York City. Address reprint requests to Dr. Cournos, New York State Psychiatric Institute, 722 West 168th St., New York, NY 10032.

Supported by NIMH grant MH-46251 and the New York State Office of Mental Health.

The authors thank Brenda Agosin, Diane Engel, M.S.W., Steve Engelmann, Arthur Harewood, Diana Hartel, Ph.D., Jim Johnson, Ph.D., Wallace Nesby, Kathline Norman, Harbert Rice, Ellie Schoenbaum, M.D., and Ezra Susser, M.D., M.P.H., for their help.

Copyright () 1993 American Psychiatric Association. filiated from social services (2), may be too disorganized to gain access to the mental health system (3), and appear to have little contact with outpatient medical settings (4). Moreover, the homeless mentally ill may not go to public shelters, which often provide on-site medical and psychiatric evaluation. A recent study found evidence of underutilization of shelters by the mentally ill living in public spaces. Of 177 persons removed from New York City streets and admitted to Bellevue Hospital for acute psychiatric care, $42 \%$ had not used public shelters during the year before admission (E. Susser, personal communication, Oct. 16, 1991).

The prevalence of HIV infection among the severely mentally ill in New York City is distressingly high. We previously reported a $5.5 \%$ seroprevalence of HIV among patients admitted to two public psychiatric hospitals in New York City (5). At a private, voluntary hospital providing acute care in New York City, 7.1\% of psychiatric inpatients were seropositive (6). Severe mental illness may increase the risk of HIV infection by contributing to indiscriminate sexual activity or drug use at times of decompensation (7-10). Prevalent substance abuse and exchanging sex for drugs, money, or 
food put persons with severe mental illness at increased risk $(8,11)$. Substance abuse may be even more prevalent among the homeless than among the domiciled mentally ill (12).

Most evidence about HIV infection among the homeless is indirect, being derived from studies which examine medical conditions that may be associated with HIV rather than the presence of HIV antibodies (13-15). Public interest in the few studies of the prevalence of HIV among the homeless has been intense. A reported HIV prevalence of $66 \%$ among 107 shelter users attending an on-site medical clinic was widely disseminated by the media and caused international alarm (R.A. Torres et al., V International Conference on AIDS, 1989). By contrast, a survey of 4,383 runaway adolescents in four states showed that $4.1 \%$ were HIV positive (R.L. Stricof et al., VI International Conference on AIDS, 1990). The striking disparity between these prevalence estimates may reflect methodologic differences as much as differences in HIV prevalence between subgroups of the homeless.

In October 1987 New York City established a link between the Homeless Emergency Liaison Project and a continuum of specialized psychiatric services, including an acute care hospital unit and an extended care state hospital ward designated for care of the homeless. The Homeless Emergency Liaison Project is a mobile outreach team that evaluates homeless men and women living on the streets who exhibit psychiatric symptoms. Designated psychiatrists are authorized to order involuntary transport to a psychiatric emergency room of persons who meet commitment criteria broadly interpreted to include grave disability as a form of dangerousness to self (16). Patients appropriate for admission are initially treated at Bellevue Hospital (17). Patients who cannot be discharged from Bellevue Hospital within approximately 28 days are transferred to a unit at Creedmoor Psychiatric Center created for extended care of the homeless mentally ill $(17,18)$. During the first year of operation of this project for the homeless, $67 \%$ of the patients admitted to Bellevue Hospital were transferred to the Creedmoor unit (19). This article reports the results of a seroprevalence study conducted among severely ill psychiatric patients admitted for extended care to the Creedmoor unit for the homeless.

\section{METHOD}

We tracked every patient between the ages of 18 and 59 years consecutively admitted to the unit for the homeless during the 18-month period from December 1989 through May 1991. Following current federal regulations for anonymous sampling (20), we obtained discard blood samples from blood drawn for routine purposes. Since samples could not be obtained for every patient at the time of admission because of refusal to permit routine blood workups, discharge of the patient before routine blood drawing, or an insufficient quantity of blood, we used any discard sample of blood drawn during the study period. We kept a roster of every patient admitted so as to obtain only a single sample of blood for each eligible patient, even if that patient had more than one admission or blood drawing during the study. This roster was not linked to HIV test results and was destroyed when all samples had been obtained.

Patients' chart number, date of birth, admission diagnosis, and number of previous psychiatric hospitalizations were obtained from their charts and recorded on the top half of a two-part form in order to obtain profiles of the eligible patients that were unlinked to their HIV status. Information on age range, gender, ethnicity, use of injected drugs, and homosexual activity among the men was obtained from the charts and recorded on the lower half of the form. Because of the small number of Asian patients admitted, Asians were grouped with Caucasians to avoid the possibility of identifying any HIV-positive Asian patient. After demographic and risk data had been obtained, the patientidentification (upper) section of the form was removed, and a six-digit bar code number was affixed to the lower section to link the blood sample with the demographic and risk data.

Patients whose blood was sampled and patients whose blood was not obtained were not significantly different in diagnosis $\left(\chi^{2}=1.71, \mathrm{df}=3, \mathrm{p}=0.63\right)$ or number of previous hospitalizations $(t=0.56, d f=17.01, p=$ 0.59 ). As a group, the 209 patients whose blood was sampled had the following clinical diagnoses: schizophrenia $(\mathrm{N}=103,49.3 \%)$, schizoaffective disorder $(\mathrm{N}=$ $90,43.1 \%$ ), affective disorders ( $\mathrm{N}=7,3.3 \%$ ), and other psychotic disorders ( $\mathrm{N}=9,4.3 \%)$. No patient was given a diagnosis of AIDS dementia. The number of known previous psychiatric hospitalizations was as follows: no hospitalizations, 25 patients (12.0\%); one hospitalization, $19(9.1 \%)$; two to five hospitalizations, 122 (58.4\%); more than five hospitalizations, 43 (20.6\%).

There were no significant differences in age or gender between the patients whose blood was obtained and those whose blood was not sampled. However, black and Hispanic patients were represented somewhat less than Caucasian and Asian patients among those whose blood was collected compared to those whose blood was not collected. Blood was not obtained for $2.1 \%$ of the Caucasian/Asian group, $8.7 \%$ of the black patients, and $15.4 \%$ of the Hispanic group $\left(\chi^{2}=7.03, \mathrm{df}=2, \mathrm{p}=0.03\right)$.

Blood samples were stored, frozen, and sent in batches of at least 25 to the New York Blood Center for HIV-1 antibody testing. Specimens that were reactive on enzyme immunoassay were tested by the Western Blot assay and then classified according to current recommendations of the Centers for Disease Control (21). Specimens were considered positive when antibodies to two of the following were detected: $\mathrm{p} 24, \mathrm{gp} 41$, and gp120/160. The presence of any single band on the Western Blot constituted an indeterminate result. The anonymous sampling did not permit obtaining and testing a second specimen from patients with indeterminate results on the first test to clarify their HIV status. 
TABLE 1. Association of HIV Infection With Demographic Characteristics, Homosexual Activity Among Men, and Use of Injected Drugs ${ }^{a}$

\begin{tabular}{|c|c|c|c|c|c|c|c|c|}
\hline \multirow[b]{3}{*}{ Variable } & \multirow{3}{*}{$\begin{array}{l}\text { Patients } \\
\text { Tested }\end{array}$} & \multirow{2}{*}{\multicolumn{2}{|c|}{$\begin{array}{c}\text { HIV- } \\
\text { Positive } \\
\text { Patients }\end{array}$}} & \multicolumn{2}{|c|}{ Unadjusted Odds Ratio } & \multicolumn{2}{|c|}{ Adjusted Odds Ratio ${ }^{b}$} & \multirow[b]{3}{*}{$\mathrm{p}$} \\
\hline & & & & \multirow{2}{*}{$\begin{array}{l}\text { Odds } \\
\text { Ratio }\end{array}$} & \multirow{2}{*}{$\begin{array}{l}95 \% \text { Confidence } \\
\text { Interval }\end{array}$} & \multirow{2}{*}{$\begin{array}{l}\text { Odds } \\
\text { Ratio }\end{array}$} & \multirow{2}{*}{$\begin{array}{c}95 \% \text { Confidence } \\
\text { Interval }\end{array}$} & \\
\hline & & $\mathbf{N}$ & $\%$ & & & & & \\
\hline Age (years) & & & & 5.74 & $1.24-26.58$ & 6.31 & $1.28-31.07$ & 0.02 \\
\hline $18-39$ & 104 & 11 & 10.6 & & & & & \\
\hline $40-59$ & 99 & 2 & 2.0 & & & & & \\
\hline Ethnicity ${ }^{c}$ & & & & 1.86 & $0.55-6.25$ & - & - & - \\
\hline Black & 93 & 6 & 6.5 & & & & & \\
\hline Hispanic & 20 & 3 & 15.0 & & & & & \\
\hline Caucasian and Asian & 90 & 4 & 4.4 & & & & & \\
\hline Sex & & & & 1.32 & $0.35-5.00$ & - & - & - \\
\hline Male & 146 & 10 & 6.8 & & & & & \\
\hline Female & 57 & 3 & 5.3 & & & & & \\
\hline Use of injected drugs & & & & 4.88 & $1.17-20.33$ & 6.67 & $1.42-31.28$ & 0.02 \\
\hline Identified & 14 & 3 & 21.4 & & & & & \\
\hline Not identified & 189 & 10 & 5.3 & & & & & \\
\hline Homosexual activity among men ${ }^{d}$ & & & & 1.57 & $0.18-13.78$ & - & - & - \\
\hline Identified & 10 & 1 & 10.0 & & & & & \\
\hline Not identified & 136 & 9 & 6.6 & & & & & \\
\hline Any risk factor & & & & 2.70 & $0.68-10.67$ & - & - & - \\
\hline Identified & 22 & 3 & 13.6 & & & & & \\
\hline Not identified & 181 & 10 & 5.5 & & & & & \\
\hline Total & 203 & 13 & 6.4 & & & & & \\
\hline
\end{tabular}

${ }^{a}$ Of the 209 individuals whose blood was tested for HIV infection, the results for six were indeterminate; therefore, data on 203 individuals were used in this analysis.

${ }^{b}$ The final logistic regression model included age, ethnicity, and use of injected drugs.

'Comparison groups for calculating the odds ratios for ethnicity were black and Hispanic versus Caucasian and Asian.

${ }^{d} \mathrm{~N}=146$, as this question pertains to male patients only.

The clinical characteristics of the patients, including admission diagnosis and number of previous psychiatric hospitalizations, constituted the group profile of the patients whose blood was sampled, unlinked to the blood specimens. Subsample seroprevalence was derived from blood test results linked with patient demographic characteristics and two HIV risk behaviors. All data were measured at either a nominal or an ordinal level; therefore, contingency table analysis was used to interpret the data. Chi-square tests were performed, and odds ratios were used to estimate relative risk. Adjusted odds ratios were derived from logistic regression coefficients. Statistical significance was expressed in exact values. The criterion for significance was set at an alpha level of 0.05 .

\section{RESULTS}

In total, we tested samples from $209(93.3 \%)$ of 224 patients admitted to the unit for the homeless during the study period. Of the 209 samples sent for antibody testing, 19 were reactive on enzyme immunoassay. Of these 19 samples, six showed indeterminate results on the Western Blot. The Centers for Disease Control indicate that individuals with reported risk whose assays are indeterminate may be in the process of conversion of their HIV status but that most often, retesting results in repeated indeterminate Western Blots (22). We concluded that the HIV status of all patients with indeterminate results was equivocal and excluded data on those six subjects, so that data on 203 patients were used in the analysis of relative risk and serostatus. The remaining 13 samples that were reactive on enzyme immunoassay were confirmed positive by Western Blot assay, for a seroprevalence of 13 of 203 , or $6.4 \%$. The distribution of positive results is shown in table 1 . No sample that was reactive on the enzyme immunoassay was negative on the Western Blot assay.

Table 1 shows that the only significant demographic variable associated with seropositivity was age. Patients between the ages of 18 and 39 accounted for $51.2 \%$ $(\mathrm{N}=104)$ of the 203 subjects and $84.6 \%(\mathrm{~N}=11)$ of the 13 positive results, a seroprevalence of $10.6 \%$ for this subsample.

The estimates of relative risk presented in table 1 were derived by using unadjusted odds ratios to compare the risk of testing positive for HIV-1 antibodies for patients with and without identified risk factors. Unadjusted odds ratios estimate relative risk for each factor without controlling for effects of other risk variables. The unadjusted odds ratios show that patients under 40 years of age were nearly six times more likely to be seropositive than patients over 40 and that patients with recorded histories of injecting drugs were almost five times as likely to be seropositive as patients with no history of such drug use on their charts. Recorded homosexual activity among the men did not predict positive HIV status in this group of patients.

Multiple logistic regression was used to analyze simultaneously the effects of age and injecting drugs, which were significant in the univariate analyses. In ad- 
dition, ethnicity, while not significantly related to seropositivity overall, was associated with age: more patients under 40 years of age than over 40 were black or Hispanic $\left(\chi^{2}=13.73, \mathrm{df}=1, \mathrm{p}=0.0002\right)$. We therefore controlled for ethnicity in the regression analysis. The adjusted odds ratios in table 1 demonstrate that for patients who were less than 40 years old, the likelihood of testing positive for HIV-1 antibodies was more than six times greater, and having a recorded history of injecting drugs independently increased the risk of HIV seropositivity more than 6.5 -fold.

When we examined the linked risk histories of the seropositive patients, we found that clinicians had identified risk behavior in three $(23.1 \%)$ of the 13 patients. Among the $10 \mathrm{HIV}$-positive men, three $(30 \%)$ engaged in known risk behaviors: one ( $10 \%)$ used injected drugs and engaged in homosexual activity, and two $(20 \%)$ were users of injected drugs. None of the three HIVpositive women had a recorded history of injecting drugs. Of the 190 seronegative patients, $12(6.3 \%)$ were identified as users of injected drugs. Among the 136 seronegative men, $10(7.4 \%)$ had histories of homosexual activity. Men with chart histories of injected drug use were significantly more likely to be HIV positive than men without such histories $\left(\chi^{2}=4.01, \mathrm{df}=1, \mathrm{p}=0.05\right)$.

\section{DISCUSSION AND CONCLUSIONS}

New York city and state governments created a single integrated program for persons who are gravely disabled, mentally ill, and living on the streets. This program provided us with a unique opportunity to conduct a seroprevalence study among a difficult-to-research group that has generated considerable public debate. While patients who could be discharged after acute care at Bellevue Hospital were not studied, most Homeless Emergency Liaison Project patients required extended care in the state hospital unit for the homeless at which we conducted this study. Our sample is therefore representative of a majority of patients removed from the streets of New York City and involuntarily hospitalized who require more intensive treatment and placement efforts. Moreover, we were able to study $93.3 \%$ of the eligible patients requiring this level of care.

The $6.4 \%$ HIV seroprevalence for these homeless patients admitted for extended care to a state hospital ward is not significantly different from the $5.5 \%$ HIV seroprevalence we previously reported for new admissions to similar state hospital wards serving predominantly domiciled patients (5). The prevalence of HIV among both the patients in the unit for the homeless and those in the general units exceeded the estimated rate of HIV infection in the general population in New York City for a corresponding time frame (5). In both groups schizophrenia was the most common diagnosis, and at least $75 \%$ of the patients had had two or more psychiatric admissions before the index hospitalization.

Women in both the unit for the homeless and the general units were as likely as men to be HIV positive. Sero- positivity among domiciled and undomiciled mentally ill women $(6.1 \%$ and $5.3 \%$, respectively) was four to five times higher than that of women delivering babies in New York City during a comparable time frame $(1.25 \%)(23)$. High-risk activity among women was inadequately identified in our studies, but previous reports suggest that heterosexual transmission may play an important role in HIV infection among severely mentally ill women (8).

We also found important differences between the patients in the unit for the homeless and those in the general units. Whereas age did not predict seropositivity in patients in the general acute care units, patients in the unit for the homeless who were 18-39 years of age were 6.5 times more likely to be infected than those aged 40 59. Among the general unit patients, black patients were six times more likely to be infected than nonblack patients. Among the homeless unit patients, ethnic differences were less pronounced. Although patients under 40 had the highest seropositivity and were more likely to be black or Hispanic than patients over 40 , our relatively small sample, in which black and Hispanic subjects were underrepresented, did not yield any clear relation between ethnicity and seropositivity.

While the seroprevalence of subgroups of the severely mentally ill may not differ widely, predictors of infection clearly diverge. For example, in contrast to both of our public hospital samples, seropositive patients at a private hospital were disproportionately male (6), a finding that mirrors the pattern of infection in the general population in New York City, where men are six times more likely to be represented among AIDS cases than women (24). Variations in sociodemographic and clinical characteristics among inpatients in different settings may be associated, in turn, with differences in high-risk behavior and the likelihood of exposure to infected sexual or needle-sharing partners. It is therefore important for investigators to describe surveyed patient groups in detail and to use caution in generalizing seroprevalence findings from one group to another.

The patient group we studied was distinctive in many respects. These patients had been living on the streets, often for extended periods of time, and many avoided the public shelter system available to the homeless in New York City. Sixty-four percent of the patients treated during the first 6 months of the Homeless Emergency Liaison Project had lived primarily in public spaces and not in shelters before admission to Bellevue Hospital (E. Susser, personal communication, Oct. 16, 1991). Furthermore, we sampled only Homeless Emergency Liaison Project patients who were gravely disabled and required treatment at an extended care facility. They were forcibly transported to emergency rooms, involuntarily admitted to an acute care hospital, and subsequently transferred for extended care to a state hospital. The group we studied, therefore, is likely to have come from the most severely impaired subgroup of the homeless mentally ill, and generalizing these findings to better-functioning homeless individuals or those who require only a short hospital stay may not be appropriate. 
The disadvantages of studying this special 70-bed unit for the homeless included the small number of new admissions during the study period, particularly among Hispanic patients. Moreover, because Hispanic patients were overrepresented among the $7 \%$ of patients from whom we could not obtain blood, the $15 \%$ HIV seropositivity for this ethnic group may be an artifact of undersampling. Previous studies have suggested that Hispanic persons are at greater risk for HIV infection than whites $(25,26)$, and special efforts should be made in future prevalence studies of the severely mentally ill to obtain a representative Hispanic sample, taking into account geographic region of origin, which is predictive of seropositivity (M.E. St. Louis et al., V International Conference on AIDS, 1989).

Other study limitations are intrinsic to the anonymous seroprevalence method (7). Among these is the need to restrict the number of variables linked to each blood sample to preserve patient anonymity. In addition, risk data were obtained from patient charts, because the anonymous study design did not permit interviews with patients. Only a minority of HIV-positive patients had risk histories on their charts, which limited our ability to make inferences about what behaviors were associated with HIV seropositivity, particularly among women. Moreover, since systematic risk assessments and psychiatric diagnoses could not be obtained by interview, we were unable to examine how psychiatric illness affects risk-taking behavior. Nevertheless, the anonymous method allowed us to obtain seroprevalence data on more than 200 street-dwelling homeless mentally ill individuals. These data represent the most complete epidemiological HIV profile of the homeless mentally ill to date and provide a basis for planning for the needs of this chronically underserved subgroup of the severely mentally ill.

Independent of the seroprevalence study, an in-depth chart review was conducted on the Creedmoor unit for the homeless that may suggest how age, ethnicity, and risk are related in this population. Compared to older patients, more of those under 40 were black or Hispanic and were more likely to use crack, a smokable analogue of cocaine (our unpublished data). Crack use is highly associated with HIV risk behavior (M.A. Chiasson et al., VI International Conference on AIDS, 1990). Crack-related high-risk behavior may account for the higher seroprevalence among patients under age 40 than among those 40 years of age or over.

Carefully conducted studies in multiple settings are needed to clarify the relation between age, ethnicity, and risk among the homeless mentally ill. Differences in psychopathology and lifestyle among subgroups of the homeless are also likely to play an important role. Street life may be associated with a pattern of drug use and sexual activity which is very different from that found in shelters for the homeless. As a result, different subgroups of the homeless may have a higher or lower prevalence of HIV infection than the group we studied.

We found that one in every 16 patients admitted to a special psychiatric unit for the homeless mentally ill was
HIV positive. Systematic procedures must be developed in psychiatric settings for inquiring about HIV-risk behavior. Aggressive investigation of risk histories is necessary for informing clinical decisions about testing and subsequent medical intervention and to stem transmission of HIV to uninfected patients. Fully $10 \%$ of the seronegative patients in our study had histories of highrisk activity on their charts. These patients should be targeted for risk reduction interventions to prevent their becoming seropositive.

Mentally ill people who live on the streets are unlikely to take advantage of traditional health care programs. Street-based interventions in which information on risk reduction and condoms are distributed have been effective with other disaffiliated populations (Y. Serrano and D. Goldsmith, V International Conference on AIDS, 1989). Similar outreach prevention efforts are urgently needed to reach the mentally ill living on the streets.

\section{REFERENCES}

1. Susser E, Goldfinger SM, White A: Some clinical approaches to the homeless mentally ill. Community Ment Health J 1990; 26: 463-480

2. Cohen NL, Putnam JF, Sullivan AM: The mentally ill homeless: isolation and adaptation. Hosp Community Psychiatry 1984; 35: 922-924

3. Fischer PJ, Breaky WR: Homelessness and mental health: an overview. Int J Ment Health 1986; 14:6-41

4. Gelberg L, Linn LS: Social and physical health of homeless adults previously treated for mental health problems. Hosp Community Psychiatry 1988; 39:510-516

5. Cournos F, Empfield M, Horwath E, McKinnon K, Meyer I, Schrage H, Currie C, Agosin B: HIV seroprevalence among patients admitted to two psychiatric hospitals. Am J Psychiatry 1991; 148:1225-1230

6. Sacks M, Dermatis H, Looser-Ott S, Perry S: Seroprevalence of HIV and risk factors for AIDS in psychiatric inpatients. Hosp Community Psychiatry 1992; 43:736-737

7. Gewirtz G, Horwath E, Cournos F, Empfield M: Patients at risk for HIV (letter). Hosp Community Psychiatry 1988; 39:13111312

8. Cournos F, Empfield M, Horwath E, Schrage H: HIV infection in state hospitals: case reports and long-term management strategies. Hosp Community Psychiatry 1990; 41:163-166

9. Sacks MH, Silberstein C, Weiler P, Perry S: HIV-related risk factors in acute psychiatric inpatients. Hosp Community Psychiatry $1990 ; 41: 449-451$

10. Sacks MH, Perry S, Graver R, Shindledecker R, Hall S: Self-reported HIV-related risk behavior in acute psychiatric inpatients: a pilot study. Hosp Community Psychiatry 1990; 41:1253-1255

11. Froner G: AIDS and homelessness. J Psychoactive Drugs 1988; 20:197-202

12. Susser E, Struening EL, Conover S: Psychiatric problems in homeless men: lifetime psychosis, substance use, and current distress in new arrivals at New York City shelters. Arch Gen Psychiatry $1989 ; 46: 845-850$

13. Nardell EA: Tuberculosis in homeless, residential care facilities, prisons, nursing homes, and other close communities. Semin Respir Infect 1989; 4:206-215

14. Centers for Disease Control, Division of Tuberculosis Control, Center for Prevention: Tuberculosis control among homeless populations. MMWR 1987; 36:257-260

15. Torres RA, Lefkowitz P, Kales C, Brickner PW: Homelessness among hospitalized patients with the acquired immunodeficiency syndrome in New York City (letter). JAMA 1987; 258: 779-780 
16. Program for Strengthening Services to the Homeless Mentally Ill in Need of Psychiatric Hospitalization. New York, New York City Health and Hospitals Corp, Sept 9, 1987

17. Marcos LR, Cohen NL, Nardacci D, Brittain J: Psychiatry takes to the streets: the New York City initiative for the homeless mentally ill. Am J Psychiatry 1990; 147:1557-1561

18. Schrage HE, Silver MA, Oldham JM: Role of the state mental health system, in Intensive Treatment of the Homeless Mentally Ill. Edited by Katz S, Nardacci D. Washington, DC, American Psychiatric Press, 1993

19. Nardacci D, Caro E, Milstein V, Schleimer H, Levy R, Erickson E, Baldwin K: Bellevue population: demographics, 61-82. Ibid

20. OPRR Reports. Washington, DC, Department of Health and Human Services Office of Protection From Research Risks, 1985, 45 CFR 46.102(f)
21. Centers for Disease Control: Interpretation and use of the Western Blot assay for serodiagnosis of human immunodeficiency virus type 1 infections. MMWR 1987; 36:509-515

22. Centers for Disease Control: Interpretation and use of the Western Blot assay for serodiagnosis of human immunodeficiency virus type 1 infections. MMWR 1989; 38(S-7):1-7

23. AIDS in New York State. Albany, New York State Department of Health, 1990

24. AIDS Surveillance Update, December 1990. New York, Department of Health AIDS Surveillance Unit, 1990

25. AMA Council for Scientific Affairs: Hispanic health in the United States. JAMA 1991; 265:248-252

26. Moran JS, Aral SO, Jenkins WS, Peterman TA, Alexander ER The impact of sexually transmitted diseases on minority populations. Public Health Rep 1989; 104:560-565 